(C) 1984. The Genetical Society of Great Britain

\title{
B CHROMOSOMES IN RATTUS FUSCIPES II. THE TRANSMISSION OF $B$ CHROMOSOMES TO OFFSPRING AND POPULATION STUDIES: SUPPORT FOR THE "PARASITIC" MODEL.
}

\author{
R. L. THOMSON
}

Dept. of Microbiology, La Trobe University, Bundoora, Victoria 3083, Australia.

Received 11.x.83

\section{SUMMARY}

Female $R$. fuscipes [in the laboratory] were observed to transmit $B$ chromosomes to their offspring at a frequency greater than expected. In two natural populations described here, this accumulation of $B s$ appears to be countered by strong selection against $B$ carrying rats which is apparent from the disproportionate loss of $+B$ individuals from the population before the breeding season.

\section{INTRODUCTION}

Over the last 30 years, observations on the transmission of $B$ chromosomes (Bs) from parent to offspring and of the comparative survival and vigour of $B$ chromosome carrying individuals have led to considerable speculation as to the method of maintenance of these apparently non-essential chromosomes in natural populations.

One school of thought considers that Bs are "parasites", their existence depending on a balance of their intrinsic accumulation mechanisms with the counter effects of reduced fertility or vigour for the "host" (e.g., in the insects Pseudococcus obscurus (Nur, 1969); Melanoplus femur-rebrum (Nur, 1977) and in Zea mays (Rhoades and Dempsey, 1973)). However, in some species the indidivuals carrying $B$ chromosomes, at least those with low numbers of $B s$, appear to have increased survival under certain conditions (e.g., in Secale cereale and Lolium perenne (Rees and Hutchinson, 1973), and again in Secale (Teoh and Jones, 1978)). Such observations have given rise to a "heterotic" model (White, 1973) where individuals with one $B$ chromosome are regarded as heterozygotes, having a selective advantage over those with no $B s$ and those with several $B s$.

Although it would appear that these two hypotheses should be easily distinguished by detailed studies of transmission of the $B s$ and the comparative survival of the $B$ chromosome carrying individuals, few studies have been reported which attempt this combined approach, particularly with mammalian $B$ chromosome systems. The present paper reports the results of such a study in Rattus fuscipes assimilis, the Australian bushrat.

\section{MAterials AND MEthods}

(i) Breeding colony

Litters were obtained from both wild caught rats and rats which had been bred in the laboratory for several generations. Chromosome constitution was determined by blood culture from adult rats and from tail culture 
or tissue culture of lung, body wall, kidney, etc. (Thomson, 1980). The progeny from 25 matings were scored for $B$ chromosome number, the litters being sampled before the tenth day after birth to avoid the complication of possible post-natal viability effects.

\section{(ii) Population study}

Aluminium box type live traps (Elliott Sci. Equip., Upwey, Vic., Aust.) were used to trap $R$. fuscipes at two sites in South Eastern Australia (fig. 1). Cambarville, an area characterised by high rainfall and temperate rainforest, carries a high density of $R$. fuscipes, and a large proportion of these have one or two $B$ chromosomes. The other site, near Anglesea, is a site of dry open woodland with a healthy understorey. The population density of $R$. fuscipes is comparatively low, but over the study period there was a higher proportion of rats with $B$ chromosomes than at Cambarville, and it is notable that some individuals carry $3 B$ chromosomes, which is unusual for this species.

Trapping was carried out monthly on a square grid (at each site) with traps arranged in rows $8 / \mathrm{m}$ apart. 100 traps were set each night. All $R$. fuscipes captured were retained for the remainder of the trapping period, and trapping was terminated when the nightly trapping success dropped below 3 rats per 100 traps. This trapping regime was favoured over one

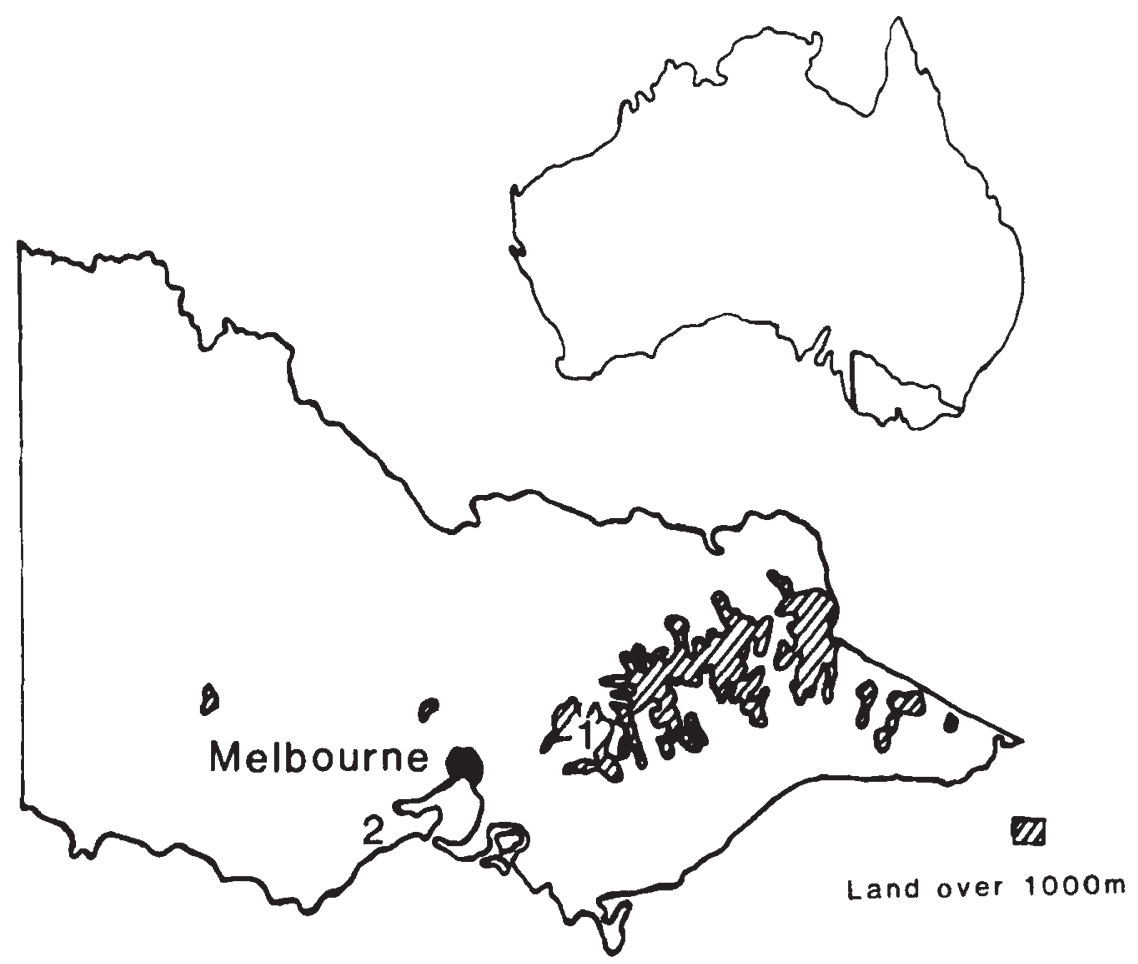

FIG. 1. Map of Victoria showing locations of the two populations sites Camberville (1) and Anglesea (2). 
where individuals are not retained but are released on the day of capture. Although the latter method would have the advantage that extra information about population size and individual movements may be gained from the capture-recapture data within each trapping period (e.g., Bishop and Hartley, 1976), it was felt that by removing rats which were captured early in the trapping period, trap-shy animals may be more likely to enter the traps on subsequent nights (MacFarlain, 1979). If this were the case, the sample would include the largest accessible population, thus reducing sampling bias.

Blood samples were taken from anaesthetised rats by the orbital sinus method of Riley (1960) using sterile capillary tubes, and cultured using standard techniques (Thomson, 1980). An assessment of age was made by measuring the width of the upper incisors. Weight and breeding condition of each rat were also noted. Rats were released at the point of capture after being individually marked by the toe clipping method described by Flowerdew (1976).

\section{RESULTS AND DISCUSSION}

\section{(i) Transmission of $B s$ from parents to offspring}

From observations of mitosis and male meiosis in $R$. fuscipes with $B$ chromosomes (Thomson et al, 1984) it is clear that there is little or no non-disjunction in premeiotic mitoses. This being the case, it is possible to gain some idea of the behaviour of $B$ chromosomes during meiosis by observing the distribution of $B$ chromosome classes amongst the progeny of directed crosses (tables 1-5).

\section{TABLE 1}

Progeny of matings of $1 B$ males $X O B$ females (three pairs, five litters)

\begin{tabular}{lrlllll}
\hline $\begin{array}{l}\text { No. of } B \text { chromosomes } \\
\text { in progeny }\end{array}$ & 0 & 1 & 2 & 3 & 4 & total \\
Observed no. of progeny & 10 & 7 & 3 & 0 & 0 & 20 \\
Expected ratio (see text) & 5 & 2 & 1 & & & 20 \\
Expected no. of progeny & 12.5 & 5 & $2 \cdot 5$ & & \\
\hline
\end{tabular}

Chi squared $=0.9(2 \mathrm{df}) \mathrm{NS}$.

Transmission frequency $=0.65$ (expect $0 \cdot 5$ ).

TABLE 2

Progeny of matings of $1 B$ females $\times O B$ males (five pairs, eight litters)

\begin{tabular}{lcccccc}
\hline $\begin{array}{l}\text { No. of } B \text { chromosomes } \\
\text { in progeny }\end{array}$ & 0 & 1 & 2 & 3 & 4 & total \\
Observed no. of progeny & 10 & 15 & 6 & 0 & 0 & 31 \\
Expected ratio (see text) & 5 & 2 & 1 & & & 31 \\
Expected no. of progeny & 19.38 & $7 \cdot 8$ & 3.82 & & \\
\hline
\end{tabular}

Chi squared $=10 \cdot 63(2 \mathrm{df})$ (significant at the $1 \cdot 0$ per cent level).

Transmission rate $=0.87$ (expect $0 \cdot 5)$. 
TABLE 3

Progeny of matings of $2 B$ males $\times O B$ females (one male, two females, two litters)

\begin{tabular}{lrrrrrc}
\hline $\begin{array}{l}\text { No. of } B \text { chromosomes } \\
\text { in progeny }\end{array}$ & 0 & 1 & 2 & 3 & 4 & total \\
Observed no. of progeny & 2 & 2 & 0 & 1 & 0 & 5 \\
Expected ratio (see text) & 25 & 20 & 14 & 4 & 1 &
\end{tabular}

Not tested statistically.

Transmission rate $=1 \cdot 0($ expect $1 \cdot 0)$.

TABLE 4

Progeny of matings of $2 B$ females $\times O B$ males (two pairs, four litters)

\begin{tabular}{lcccccc}
\hline $\begin{array}{l}\text { No. of } B \text { chromosomes } \\
\text { in progeny }\end{array}$ & 0 & 1 & 2 & 3 & 4 & total \\
Observed no. of progeny & 3 & 4 & 2 & 2 & 0 & 11 \\
Expected ratio (see text) & 25 & 20 & 14 & 4 & 1 & \\
Expected no. of progeny & 4.30 & 3.44 & 2.42 & 0.66 & 0.18 & 11 \\
\hline
\end{tabular}

Not tested statistically.

Transmission rate $=1 \cdot 27(\operatorname{expect} 1 \cdot 0)$.

TABLE 5

Progeny of matings of $1 B$ females $\times 1 B$ males (three pairs, five litters)

\begin{tabular}{lcccccc}
\hline $\begin{array}{l}\text { No. of } B \text { chromosomes } \\
\text { in progeny }\end{array}$ & 0 & 1 & 2 & 3 & 4 & total \\
Observed no. of progeny & 3 & 7 & 6 & 2 & 0 & 18 \\
Expected ratio (see text) & 26 & 20 & 14 & 4 & 1 & \\
Expected no. of progeny & 7.03 & 5.62 & 3.94 & 1.13 & 0.28 & 18 \\
\hline
\end{tabular}

Not tested statistically.

Transmission rate $=1 \cdot 39($ expect $1 \cdot 0)$.

It is obvious from these results that the $B$ chromosomes do not behave in the same manner as the members of the normal complement. Normal meiotic behaviour (where homologues pair, separate, and move to opposite first division poles, then dyads split at the centromere during the second division and each replicate chromosome moves to opposite poles) cannot account for the $2 B$ gametes produced by $1 B$ individuals (tables 1 and 2 ) or for the $3 B$ gametes produced by $2 B$ individuals (tables 3 and 4 ).

One simple hypothesis which can qualitatively account for the distribution of $B$ chromosomes in progeny in these results is that $B$ chromosomes move at random at each meiotic division. Such random distribution would be achieved if

(a) Bs did not pair at first division and moved randomly to either pole (evidence from $R$. fuscipes male meiosis suggests that is the case; Thomson, 1984). 
(b) $\boldsymbol{B}$ dyads at second division separated early and each replicate moved polewards at random (rather than to opposite poles).

As can be seen from table 1 , the distribution of $B$ chromosomes in the progeny of $1 B$ male $\times 0 B$ female matings is consistent with this hypothesis. However, table 2 shows that the reciprocal cross produces an excess of $B$ chromosome progeny. The average transmission rate for this cross is 0.87 , compared to the expected value of $0 \cdot 5$. This suggests that a "meiotic drive" mechanism is operating in females. Consistent with this hypothesis is the observation that the $2 B$ female $\times 0 B$ male matings also have a high average transmission frequency, whilst for $2 B$ male $\times 0 B$ female mating the transmission frequency is as expected (admittedly with small sample sizes). Similarly, the $1 B \times 1 B$ cross (table 5 ) has an average frequency of $B$ chromosomes in the progeny of $1 \cdot 39$, which is again consistent with an increased transmission frequency, although in this cross the differences between males and females are masked.

These results can be compared with those of Yosida (1978), who observed the transmission of $B$ chromosomes in Rattus rattus. His results, although not conclusive in themselves, also suggest an excess of $B$ chromosome progeny in the offspring from $B$ chromosome females. Thus these related species may have a similar mode of $B$ chromosome inheritance, conforming to a pattern of random segregation at both meiotic divisions in the male, but with a meiotic drive mechanism operating in the female, leading to an accumulation of $B$ chromosomes in oocyte nucleii.

It should be pointed out that the term "meiotic drive" is being used rather loosely here. True meiotic drive systems seem to cause their distorted segregation of allele frequencies through the production of dysfunctional gametes. For example, Segregation-Distorter (S.D.) in Drosophila melanogaster involves the directed breakdown of spermatids (Peacock et al., 1972), and the T-system in the mouse is thought to operate through a differential loss of mature sperm, but may be directed by events shortly after meiosis when the spermatids are interconnected (Braden, 1972). Evidence of true meiotic drive of $B$ chromosomes in a mammal has been reported by Gileva and Chebotar (1979) in the female hoofed lemming. Of course, female gametogenesis presents the opportunity for accumulation as there is a controlled loss of half the primary oocytes via the polar body. Gileva and Chebotar give cytogenetical evidence that $B$ chromosome univalents in this species (as in Myrmeleotettix maculatus, Hewitt, 1976) are directed away from the polar body and become incorporated into the secondary oocytes. They also show that some accumulation occurs in female premeiotic mitoses. It is envisaged that the mechanism of accumulation of $B$ chromosomes in females of the two Rattus species is similar to that in the hoofed lemming. However, in $R$. fuscipes, there is no real evidence to show that the possible distortion of $B$ chromosome transmission in females is not due to differential zygotic mortality. Warneke (1971) found that $R$. fuscipes in his field population had a pre-natal loss of about 23 per cent (13.5 per cent attributable to loss of ova, and 9.5 per cent attributable to zygotic death). In the light of the results presented here, a further study to determine whether there is differential mortality of $B$ carrying and $0 B$ embryos would be interesting.

Transmission has been studied in few mammalian $B$ chromosome systems. However, Patton's (1977) study of male meiosis in Perognathus baileyi 
does give some insight into the mode of inheritance of $B s$ in this species, and shows that this system is rather different from that of the two Rattus species discussed above. In $2 B$ males, $B$ chromosome bivalents are seen in about half the first metaphase spreads, and in individuals with more $B$ chromosomes, multivalents are often observed. Such a situation would make random segregation less likely than if the $B s$ are regularly unpaired at first metaphase, as in Rattus. As well as this, no second metaphase cells are seen with more $B$ chromosomes than are found in the somatic cells, suggesting that the centromeres of $B$ dyads are not separating earlier than the centromeres of the $A$ chromosome dyads. If this so, random segregation in second division is unlikely. These two points, along with the possibility of meiotic drive in the male, suggest that the general mode of transmission of $B$ chromosomes is radically different from that of the two Rattus species.

\section{(ii) Survival of $B$ chromosome-carrying $R$. fuscipes in natural populations}

The population dynamics of $\boldsymbol{R}$. fuscipes in South Eastern Australia has been investigated by several workers, including Warneke (1971) and Robinson (1976). At the two study sites (Cambarville and Anglesea) the populations behaved similarly to the populations described by those workers. The rats have a well defined breeding season, with juveniles entering the trappable population from mid summer. In autumn, there is general dispersal of these immature rats with, possibly, some mortality. Over winter, there appears to be little further dispersal and little mortality. In spring the males disperse. After the breeding season (which may extend through to late summer), there is a general loss of adult rats of both sexes from the population, presumably due to mortality. By autumn, very few adult rats are known to be alive. Some females (less than 10 per cent) survive until the next breeding season, but virtually no males survive into their second winter. Warneke (1971) found that all surviving females produce litters in the breeding season after their birth, and indeed some may produce two or three litters. Very few individuals of either sex breed in the season of their birth.

The existence of such a rigid pattern of reproduction allows us to estimate the numbers of animals lost from a defined area before they have the opportunity to breed. Rats which had been trapped previously, but were not known to be alive in the November trapping period were assumed to be absent from the breeding population on each of the two sites (fewer than 3 per cent of rats on either site conceived before the November trapping period in any year of the study).

A breakdown of the data on loss of individuals from the populations for $0 B$ and $B$ chromosome groups does show some important differences. These results are presented in tables 6 and 7 .

Table 6 shows that loss of rats from the populations is independent of sample site, or of sex. That is, the pattern of loss could be considered to be the same for both sexes at both sample sites.

Partitioning these results (table 7) shows that for both sexes at Cambarville there is a significantly greater loss of rats with $B$ chromosomes before the breeding season than of rats without $B s$ (table 7 parts a and b). Anglesea males (part c) also have a significant difference for the loss of $B$ chromosome rats and $0 B$ rats. The Anglesea females do not show a significant difference 
TABLE 6

Numbers of rats lost from the population: males and females for both Anglesea and Cambarville compared

\begin{tabular}{|c|c|c|c|c|}
\hline & \multicolumn{4}{|c|}{ Numbers of rats lost } \\
\hline & \multicolumn{2}{|c|}{ Before breeding* } & \multicolumn{2}{|c|}{ After breeding $\dagger$} \\
\hline & $O B$ & $+B$ & $O B$ & $+B$ \\
\hline Cambarville males & 8 & 12 & 36 & 16 \\
\hline Cambarville females & 8 & 15 & 25 & 14 \\
\hline Anglesea males & 11 & 16 & 30 & 15 \\
\hline Anglesea females & 3 & 6 & 11 & 5 \\
\hline
\end{tabular}

Chi squared $=2 \cdot 55,9 \mathrm{df}, \operatorname{Pr}>0.05$.

* Rats lost before breeding are defined as those which are trapped on the grids but are not known to be alive in the breeding season after their birth (November or later).

$\dagger$ Rats lost after the breeding season are defined as those which are known to be alive at any time during the breeding season.

TABLE 7

Numbers of $B$ chromosome $(+B)$ and $O B$ individuals lost from the population

\begin{tabular}{lcccc}
\hline & \multicolumn{4}{c}{ Numbers of individuals lost } \\
\cline { 2 - 5 } \multicolumn{1}{c}{ Sample } & $\begin{array}{c}B \text { chromosome } \\
\text { constitution }\end{array}$ & $\begin{array}{c}\text { Before } \\
\text { breeding* }\end{array}$ & $\begin{array}{c}\text { After } \\
\text { breeding }\end{array}$ & $\begin{array}{c}\text { Chi squared } \\
\text { value }\end{array}$ \\
\hline (a) Cambarville males & $0 B$ & 8 & 36 & 5.19 \\
& $+B$ & 12 & 16 & $\operatorname{Pr}<0.05$ \\
(b) Cambarville females & $0 B$ & 8 & 25 & 4.99 \\
& $+B$ & 15 & 14 & $\operatorname{Pr}<0.05$ \\
(c) Anglesea males & $0 B$ & 11 & 30 & 4.62 \\
& $+B$ & 16 & 15 & $\operatorname{Pr}<0.05$ \\
(d) Anglesea females & $0 B$ & 3 & 11 & $1.67 \ddagger$ \\
& $+B$ & 6 & 5 & $\operatorname{Pr}>0.05$ \\
(e) Total & $0 B$ & 30 & 102 & 18.01 \\
& $+B$ & 49 & 50 & $\operatorname{Pr}<0.001$
\end{tabular}

* Rats lost before breeding are defined as those which have been trapped on the grids but are not known to be alive in the breeding season after their birth (November or later).

$\dagger$ Rats lost after the breeding season are defined as those which are known to be alive during the breeding season.

$\ddagger$ The chi squared test on the $2 \times 2$ contingency table for part $(d)$ is corrected for continuity.

between chromosome groups, but it is clear from the analysis presented in table 6 that the pattern observed is consistent with that for Anglesea males and for the Cambarville population.

Because the data are not significantly heterogeneous, it is possible to estimate from the lumped data the percentage loss of $B$ chromosome and $O B$ individuals from the populations before breeding. This is presented in 
table $7(\mathrm{e})$. It can be seen that $30 / 132=22 \cdot 7$ per cent of rats without $B$ chromosomes are lost before breeding, whilst $49 / 99=49.5$ per cent of rats with $B$ chromosomes are lost $(\operatorname{Pr}<0.001$ for the contingency chi squared test). Thus, rats with $B$ chromosomes are approximately twice as likely to be lost from the population before breeding as $0 B$ rats.

Loss of individuals from a population is a complex statistic. Whilst it is possible to envisage that a differential mortality is a major cause, differential migration could be occurring, with more $B$ chromosome rats than $0 B$ rats leaving the sampling site before breeding season, and/or fewer $B$ chromosome individuals entering the population during the breeding season. For some mammalian species, it is known that dispersing individuals may have a different genotype from those animals resident in the population (as determined by electrophoresis, e.g., in Microtus, (Myers and Krebs, 1971; Krebs et al., 1976)). However, there is considerable debate as to whether the genetic variation is associated with the cause or the effect of dispersal (see Liddicker, 1978; Tamarin, 1978). It was not possible in the present study to investigate the frequencies of $B$ chromosomes in dispersing animals, one major problem being the inability to obtain karyotypes of several animals which were captured only once (i.e., transients). In theory $B$ chromosomes could affect dispersal behaviours directly by the action of genes located on the $B$ chromosomes themselves, either autonomously or via effects on genes on the $A$ chromosomes. The $C$ band-positive nature of the $B$ chromosome (Yosida and Sagai, 1973; Thomson et al., 1984), however, suggests that much of it is constitutively heterochromatic and thus probably carries relatively few active genes, although this requires confirmation.

Alternatively, $B$ chromosomes could act indirectly simply through the presence of the extra (possibly non-coding) DNA. For example, a $B$ chromosome correlated phenotype effect such as slowed development may put many $B$ carrying individuals into a class of individuals prone to dispersal. Whilst the existence of such a class is unknown in rats, it has been reported that in voles (Microtus), dispersers tend to be smaller and reach sexual maturity at lower weights than do residents (Myers and Krebs, 1971; Krebs et al., 1976; Tamarin, 1977). Beacham suggests that from his data on M. townsendii dispersal and growth rate are closely related (Beacham, 1979), and that dispersal of smaller voles is caused by aggressive interactions with larger voles. The larger voles are able to remain as residents. In $R$. fuscipes, there is no information as to whether individuals which remain as residents are different phenotypically from those which disperse. As well, laboratory data show that $B$ chromosome effects on growth rate appear to be negligible under standardised conditions (Thomson, 1980). Thus, no firm conclusions can be made about the $B$ chromosome contribution to the migration component of loss from $R$. fuscipes populations.

Nevertheless, even if the differential loss of $B$ carrying rats from the population is only partly due to differential mortality, the final outcome is still of considerable importance: a lower proportion of $B$ carrying rats is present on the sample site at breeding season than was born onto the site in the previous season. For females this means that a lower proportion of $B$ chromosome rats will breed than expected on the basis of the proportion of $B$ carrying rats in the population at the end of the previous breeding season (since almost all females produce litters, Warneke, 1971). For males 
there is no information available about variation in breeding success, but on the basis of opportunity to breed, the conclusion reached is the same as for females.

This implies that there is effective selection against the $B$ carrying individuals. For Mendelian systems, selection pressures of this strength would rapidly eliminate the allele being selected against. Thus, for the frequency of $B$ chromosomes in the population to remain relatively constant, an excess of $B$ carrying rats must be present in the overall population at the time when young rats enter the trappable population. The results of the breeding studies show that there is indeed a higher transmission of $B$ chromosomes to offspring than expected (at least via the female), and this should produce an excess of $B$ carrying juveniles.

Ultimately a model could be constructed using the transmission frequencies calculated from laboratory crosses, in order to estimate the excess of $B$ carrying juveniles which may be produced by a breeding population with a particular frequency of $B s$ amongst the parents. However the results presented here are based on too few individuals to give accurate estimates of transmission frequencies.

Whether the accumulation of $B s$ in natural populations is strong enough to balance the observed loss before the breeding season of individuals carrying $B$ chromosomes, cannot be determined without further studies. A selective advantage for individuals with $B$ chromosomes in the first month after birth could contribute to the excess of juveniles with $B s$ which enter the trappable population. It is clear, however, from the numbers of individuals carrying $B$ chromosomes which are lost from the population before breeding, that an overall heterotic effect of the $B s$ is not possible. Thus the results presented here favour the "parasitic" model of $B$ chromosome maintenance in natural populations of Rattus fuscipes assimilis.

Acknowledgments. Part of this work was undertaken whilst I was in receipt of a LaTrobe University Postgraduate Scholarship. All trapping and handling of $R$. fuscipes was performed under permit as issued by the Fisheries and Wildlife Division of the Ministry for Conservation, Victoria. I am especially grateful to Drs Neil Murray and Mike Westerman for valuable discussions throughout the project and for reading this manuscript.

\section{REFERENCES}

BEACHAM, T. D. 1979. Size and growth characteristics of dispersing voles (Microtis townsendii). Oecologia, 42, 1-10.

BISHOP, J. A. AND HARTLEY, D. J. 1976. The size and age structure of rural populations of Rattus norvegicus containing individuals resistant to the anticoagulant poison, warfarin. J. Anim. Ecol., 45, 623-646.

BRADEN, A. W. 1972. T-locus in mice: segregation distortion and sterility in the male. In Beatty, R. A. and Gluecksohln-Waelsch, S. (eds.) The Genetics of the Spermatozoan Genetics Dept., Edinburgh, pp. 289-305.

FLOWERDEW, J. R. 1976. Ecological Methods. Mamm. Rev., 6, 123-160.

GILEVA, E. A. AND CHEBOTAR, N. A. 1979. Preovulatory stages of gametogenesis, the sex chromosome and $B$ chromosome behaviour at female meiosis of Dicrostonyx torquatus Pall. Tsitologiya, 21, 798-801.

HEWITT. G. 1976. Meiotic drive for $B$ chromosomes in the primary oocyte of Myrmeleotettix maculatus (Orthoptera: Acrididae). Chromosoma, 56, 381-391.

KREBS, C. J., WINGATE, I., LEDUC, J., REDFIELD, J. A., TAITT, M. AND HILBORN, R. 1976. Microtis population biology: dispersal and fluctuations of M. townsendii. Can. J. Zool., 54, 79-95. 
LIDDICKER, W. Z. 1978. Regulation of numbers in small animal populations: historical reflections and a synthesis. In Populations of Small Mammals under Natural Conditions. Pymatuning Laboratory of Ecology Special Publication No. 5. Pittsburgh University Press, pp. $122-141$.

MACFARLAIN, M. A. 1978. Mammal survey techniques in Victorian forests. Bull. Aust. Mamm. Soc., 6, 51.

MYERS, J. H. AND KREBS, C. J. 1971. Genetic, behavioural and reproductive attributes of dispersing field voles Microtis pennsylvanicus. Ecol. Monogr., 44, 53-78.

NUR, U. 1969. Harmful $B$ chromosomes in a mealy-bug: additional evidence. Chromosoma, $28,280-297$.

NUR, U. 1977. Maintenance of a "parasitic $B$ chromosome in the grasshopper Melanoplus femur-rubrum. Genetics, 87, 499-512.

PATtON, J. L. 1977. B chromosome systems of the pocket mouse Perognathus baileyi: Meiosis and $C$ band studies. Chromosoma, 60, 1-14.

PEACOCK, W. J.. TOKUYASU, K. AND HARDY, R. N. 1972. Spermiogenesis and meiotic drive in Drosophila. In Beatty, R. A. and Gluecksohln-Waelsch, S. (eds.) The Genetics of the Spermatozoon. Genetics Dept. Edinburgh University, pp. 247-268.

REES, H. AND HUTCHINSON, J. 1973. Nuclear DNA variation due to $B$ chromosomes. Cold Spring Harbour Symp. Quant. Biol., 38, 175-182.

RHOADES. M. A. AND DEMPSEY. E. 1973. On the mechanism of chromatin loss induced by the $B$ chromosomes of maize. Genetics, 71, 73-96.

RILEY, V. 1960. Adaptation of the orbital bleeding technique to rapid serial blood studies. Proc. Soc. exp. Biol. N.Y., 104, 751-754.

ROBINSON, A. C. 1976. Some aspects of the population ecology of the bushrat Rattus fuscipes. PhD. Thesis, Monash University, Victoria, Aust.

TAMARIN, R. H. 1978. A defence of single-factor models of population regulation. Populations of Small Mammals Under Natural Conditions. Pymatuning Laboratory of Ecology Special Publication No. 5. Pittsburgh University Press, pp. 159-162.

TEOH, S. B. AND JONES, R. N. 1978. B chromosome selection and fitness in Rye. Heredity, 41, 35-48.

THOMSON, R. L. 1980. Cytogenetical and Population Studies of B chromosomes in Rattus fuscipes (Rodentia: Muridae). MSc. Thesis, LaTrobe University, Australia.

THOMSON, R. L., WESTERMAN, M. AND MURRAY, N. D. 1984. B chromosomes in Rattus fuscipes I: mitotic and meiotic chromosomes and the effects of $B$ chromosomes on chiasma frequency. Heredity, 52, 355-362.

WARNEKE, R. M. 1971. Field Studies of the Bushrat Rattus fuscipes. Wildl. Contr. Victoria, $14,1-114$.

WHITE, M. J. D. 1973. Animal cytology and Evolution. Third Edn. Cambridge Univ. Press.

YOSHIDA, T. H. 1977. Supernumerary chromosomes in the black rat (Rattus rattus) and their distribution in three geographical variants. Cytogenet. Cell Genet., 18, 149-159.

YOSHIDA, T. H. 1978. Some genetic analysis of supernumerary chromosomes in the black rat in laboratory matings. Proc. Jap. Acad. 54, Ser. B., 440-445. 